\title{
Impact on clinical outcome of secondary brain insults during the neurointensive care of patients with subarachnoid haemorrhage: a pilot study
}

\author{
P Enblad, L Persson
}

\begin{abstract}
Objective-To analyse the occurrence and influence on outcome of secondary brain insults during neurointensive care of patients with subarachnoid haemorrhage. Methods-Sixty one seriously ill patients with subarachnoid haemorrhage with a poor neurological grade, acute hydrocephalus, or intraventricular and/or intracerebral haemorrhages, who were referred as emergency cases to the neurosurgical intensive care unit during 1990 and 1991 , comprised the study patients.
\end{abstract}

Results-The follow up performed according to the Glasgow outcome scale 14 months (median) later showed 23 patients with good recovery $(38 \%), 11$ with moderate disability $(18 \%)$, seven with severe disability (11\%), and two in a vegetative state $(3 \%)$; 18 patients had died $(30 \%)$. Clinical outcome was significantly related to the CT modified Hunt and Hess grade $(P=0.006)$. In total, 164 secondary brain insults (potentially avoidable factors) of various types were seen at the unit during the first seven days after the haemorrhage. Patients with a favourable outcome had significantly fewer secondary insults than patients with an unfavourable outcome $(P=0 \cdot 0008)$. The occurrence of insults in each patient was related to the neurological grade (CT modified Hunt and Hess grade, $P=0.05$ ). Multivariate analysis with the CT modified Hunt and Hess grade and the number of secondary brain insults during the first week as explanatory variables and favourable outcome as the dependent variable, showed that the number of complications was a significant independent predictor of favourable outcome $(\beta=$ $-0.38, \operatorname{SE}(\beta)=0.17, P=0.03)$, whereas the CT modified Hunt and Hess grade did not reach significance $(\beta=-1 \cdot 2, \operatorname{SE}(\beta)=$ $0 \cdot 81, P=0 \cdot 14$ ).

Conclusions-The clinical outcome after subarachnoid haemorrhage is at least partly determined by the number of secondary insults. Therefore, vigorous attempts should be made to avoid all events that may potentially increase the risk of secondary cerebral ischaemia. Prospective studies must be initiated to define the role of "priming" of the brain and the impact of specific individual secondary insults in patients with subarachnoid haemorrhage.
(F Neurol Neurosurg Psychiatry 1997;62:512-516)

Keywords: subarachnoid haemorrhage; neurosurgical intensive care; secondary brain insults

Early studies in the 1970 s of patients who had talked and died after head injury disclosed that secondary insults were common and had a devastating impact on outcome. ${ }^{12}$ Since then, several studies have emphasised the importance of avoiding secondary insults in patients with head injury, ${ }^{3-8}$ both those occurring during the initial management ${ }^{910}$ or later during intensive care. ${ }^{11} 12$ However, the precise occurrence and clinical relevance of intracranial and systemic complicating factors that appear after subarachnoid haemorrhage during neurosurgical intensive care has, to our knowledge, not previously been reported, although the concept of "avoidable factors" should also apply to subarachnoid haemorrhage. Obviously, unfavourable outcome is, in many cases, caused directly by bleeding or rebleeding, or delayed ischaemic deterioration (vasospasm). Delayed ischaemic deterioration is, in our opinion, a clinically indistinct entity and the development of cerebral infarction is probably the result of a complex pathophysiological process involving factors other than arterial vasospasm. This is illustrated by the fact that there are patients with severe angiographic vasospasm without ischaemic symptoms or CT demonstrable infarcts. On the other hand, patients with an essentially normal angiography may develop delayed ischaemic symptoms and infarcts. Evidently, factors other than vasospasm must be involved in the development of ischaemia. From clinical experience, it is clear that patients with secondary intracranial hypertension due to intracerebral or intraventricular haematoma or to acute hydrocephalus are more prone to develop delayed ischaemia and infarcts. Work performed in animal models has clearly demonstrated that the effects of cerebral ischaemia are aggravated by factors such as systemic hypotension, hypoxaemia, and pyrexia. Furthermore, both clinical and experimental findings suggest that the ictus (a transient abrupt intracranial hypertension ${ }^{1314}$ ) may increase the vulnerability of the brain to later focal ischaemic impacts. ${ }^{15}$

With this background, we performed a retrospective pilot study of severely ill patients with subarachnoid haemorrhage with the aim of collecting all available data on secondary 
Table 1 Clinical outcome according to the Glasgow outcome scale (GOS) in relation to the CT modified Hunt and Hess grade

\begin{tabular}{|c|c|c|c|c|c|c|}
\hline \multirow[b]{2}{*}{ GOS } & \multicolumn{6}{|c|}{ CT modified Hunt and Hess grade } \\
\hline & $I$ & II & $I I I$ & $I V$ & $V$ & Total \\
\hline $\begin{array}{l}\text { Good recovery } \\
\text { Moderate disability } \\
\text { Severe disability } \\
\text { Vegetative state } \\
\text { Death }\end{array}$ & & 1 & $\begin{array}{r}11 \\
1 \\
1 \\
1\end{array}$ & $\begin{array}{r}11 \\
9 \\
6 \\
2 \\
9\end{array}$ & 1 & $\begin{array}{r}23 \\
11 \\
7 \\
2 \\
18\end{array}$ \\
\hline Total & & 1 & 14 & 37 & 9 & 61 \\
\hline
\end{tabular}

events and making a preliminary evaluation of the possible influence on outcome.

\section{Material and methods}

PATIENTS AND CLINICAL MANAGEMENT

The investigation included seriously ill patients with subarachnoid haemorrhage, defined as patients with impaired consciousness; patients with agitation, severe headache, and often high fluctuating blood pressure in combination with extensive amount of subarachnoid blood on CT; or the presence of intraventricular or intracerebral blood or acute hydrocephalus. Almost all patients were referred within 24 hours to the neurosurgical intensive care unit during 1990 and 1991. The diagnosis of aneurysmal subarachnoid haemorrhage was confirmed by angiography. Patients dying before angiography could be performed were included, when their medical history and CT findings clearly suggested aneurysmal subarachnoid haemorrhage or its cause was established by necropsy. There were 61 consecutive patients, 36 women between 30 and 70 years of age $($ mean $=53$, median $=$ 54 ) and 25 men between 32 and 64 years of age $($ mean $=49$, median $=51)$. All patients were fitted with a ventriculostomy.

Intensive neurological and physiological monitoring with aggressive therapy of derangements was the general philosophy at the unit to avoid or minimise secondary insults. The treatment goals were an intracranial pressure (ICP) below $20 \mathrm{~mm} \mathrm{Hg}$ and a cerebral perfusion pressure (CPP) above 60 $\mathrm{mm} \mathrm{Hg}$, normal blood gases, normal fluid and electrolyte balance, normotension, temperature below $38^{\circ} \mathrm{C}$, and blood glucose of 5-10

Table 2 Secondary brain insults during the first week after subarachnoid haemorrhage in 55 patients

\begin{tabular}{llr}
\hline Insults & Definitions & No \\
\hline (1) Increased ICP & $\geqslant 25 \mathrm{~mm} \mathrm{Hg}$ & 14 \\
(2) Inadequate CPP & $\leqslant 70 \mathrm{~mm} \mathrm{Hg}$ & 48 \\
(3) Systemic hypotension & Systolic blood pressure $\leqslant 90 \mathrm{~mm} \mathrm{Hg}$ & 5 \\
(4) Hypoxaemia & pO $\leqslant 10 \mathrm{kPa}$ & 3 \\
(5) Pyrexia & Body temperature $\geqslant 39^{\circ} \mathrm{C}$ & 14 \\
(6) Hyperglycaemia & Blood glucose $\geqslant 15 \mathrm{mmol} / 1$ & 2 \\
(7) Clinical ischaemic deterioration & Fluctuating global and/or focal signs & 12 \\
(8) Tentorial herniation & Pupillary dilatation & 6 \\
(9) Seizures & Clinical signs & 8 \\
(10) Focal ischaemic lesion & CT findings (low attenuated areas) & 12 \\
(11) Haematoma & CT findings & 4 \\
(12) Rebleeding & Clinical signs and CT findings & 3 \\
(14) Ureningitis & Cultures & 1 \\
(15) Septicaemia & Cultures & 10 \\
(16) Wound infection & Cultures & 5 \\
(17) Pneumonia & Cultures & 3 \\
(18) Myocardial infarction & Chest radiographs & 12 \\
& ECG, enzymes & 2 \\
& & Total 164
\end{tabular}

*For complications 1-6, these deviations had to be recorded for at least three consecutive hours and a maximum of one insult per day of each specific type was taken into consideration. $\mathrm{mmol} / \mathrm{l}$. Early surgery was performed, except in patients in a poor neurological condition. All patients received nimodipine. Delayed ischaemic deterioration was treated with hypertensive, hypervolaemic, and haemodilutional therapy. Unconscious patients were kept on artificial ventilation.

\section{PROGNOSTIC FACTORS ON ADMISSION}

A battery of clinical and radiological characteristics of each patient available on admission were assessed. These were age, sex, seizures in the early acute phase, CT modified Hunt and Hess grade, ${ }^{16-18}$ presence of hemiparesis, and presence or absence of any relevant intercurrent disease irrespective of type, as well as hypertension specifically (pre-existing antihypertensive treatment). CT modified Hunt and Hess grade denotes that patients in Hunt and Hess grades I-III are assigned to the next less favourable grade if their CT was in Fisher groups 3 or $4 .{ }^{17}$

Table 1 gives the patient characteristics on admission according to the CT modified Hunt and Hess grading system. Brain CT was categorised as Fisher group 4 (intraventricular or intracerebral clots) in 54 of the 61 patients. Fourteen patients had a history of seizures during the early acute phase and 17 had hemiparesis on admission. Twenty six patients had one or more pre-existing intercurrent diseases and arterial hypertension had been previously diagnosed in 10 patients. Of the 51 patients who underwent angiography, the aneurysm was located in the internal carotid artery in 10, anterior cerebral artery/anterior communicating artery in 17 , middle cerebral artery in 14 , and in the vertebrobasilar circulation in one, and nine patients had multiple aneurysms.

SECONDARY BRAIN INSULTS DURING NEUROINTENSIVE CARE

All insults occurring during the first week after the subarachnoid haemorrhage were registered according to defined criteria (table 2). The choice and definitions of insults was based on our clinical impression of the ordinary occurrence and the supposed clinical impact. The total number of complications occurring was assessed and the relation between the number of complications and clinical outcome was analysed. Six patients with final brain herniation on admission or during the first day at the department were not included in this analysis because they were not at any risk of developing complications during neurointensive care, as complications occurring after final brain herniation but before cerebral death was legally declared were not included. To present total management results as far as possible, these patients were not excluded in the analysis of the overall results.

\section{OUTCOME MEASURES}

The Glasgow outcome scale was used to assess the clinical outcome of the patients. ${ }^{19}$ Good recovery and moderate disability according to the Glasgow outcome scale were defined as favourable outcome and severe disability, vegetative state, and death as unfavourable. The 
Figure 1 Relation between clinical outcome (favourable or unfavourable) and mean number of secondary brain insults occurring during the first week after subarachnoid haemorrhage.

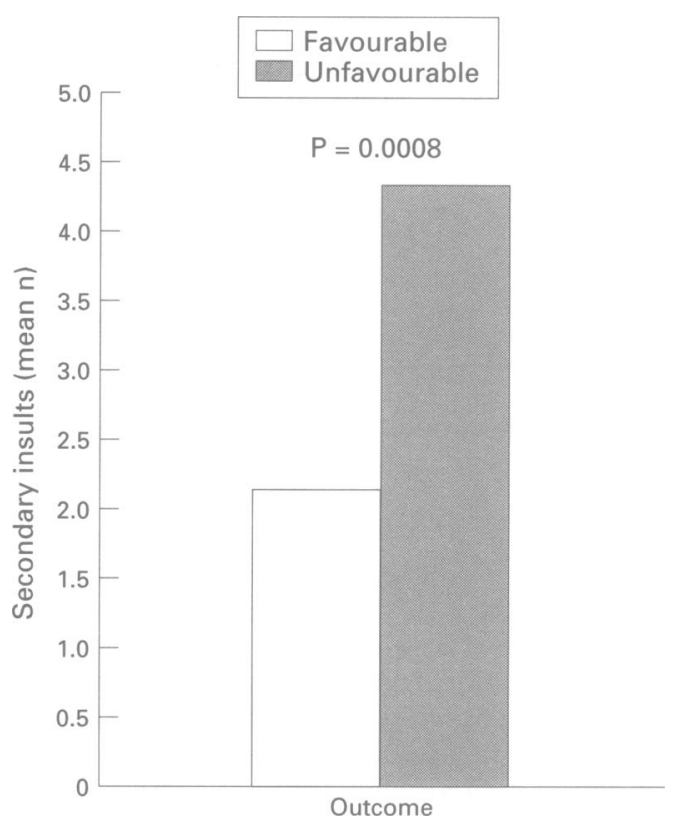

evaluation was performed by one of the research nurses at the neurointensive care unit. The median follow up was 14 months (average $13 \cdot 8$, range $2-30$ ).

\section{STATISTICAL METHODS}

A standard $\chi^{2}$ test for homogeneity was employed for categorical variables in the univariate analysis. In the case of continuous variables, a standard $t$ test was used to test differences between groups. In the multivariate analyses, a logistic regressional model was used. In this model, it is assumed that the logarithm of the odds of "favourable outcome" is a linear function of explanatory variables. The model enables the estimation of the effects of variables after adjustments for the effects of other variables. In the multiple analysis, only variables which had displayed significant differences in the univariate analysis were included.

\section{Results}

OVERALL OUTCOME

The overall outcome in this selected group of patients with serious subarachnoid haemorrhage was classified as good recovery in 23 patients $(38 \%)$, moderate disability in 11 $(18 \%)$, severe disability in seven $(11 \%)$, vegetative state in two (3\%), and death in 18 $(30 \%$; table 1$)$. Of the 10 patients who died before discharge, six showed signs of tentorial herniation on admission or during the first day in the unit. In the remaining four patients, death was caused by cardiac arrest in one, cerebral infarction in one, and rebleeding in two. Of the eight patients who died after discharge, death was related to the subarachnoid haemorrhage in all but one who died of a heart attack (discharged without sequele).

Surgery for aneurysm was performed in $\mathbf{5 0}$ patients and usually at an early stage (median = day 2). One aneurysm was considered inoperable and 10 patients were not operated on because of poor neurological condition.

PROGNOSTIC FACTORS ON ADMISSION

In the univariate analyses, the outcome was strongly correlated to the CT modified Hunt and Hess grade $\left(\chi^{2}\right.$ test, CT modified Hunt and Hess grades I-III/IV-V: favourable/ unfavourable outcome, $P=0.006$; table 1 ). Other admission variables such as age, sex, intercurrent disease, arterial hypertension, hemiparesis, and aneurysm location showed no significant relation with clinical outcome.

SECONDARY BRAIN INSULTS DURING

NEUROINTENSIVE CARE

In total, we identified 164 insults during the first seven days after the haemorrhage (mean $=3$, range $0-11$, median $=3$ ), according to our definitions (table 2). The mean number of complications was 1.9 in patients in CT modified Hunt and Hess grades I-III and 3.4 in patients in CT modified Hunt and Hess grades IV-V $\left(\chi^{2}\right.$ test, CT modified Hunt and

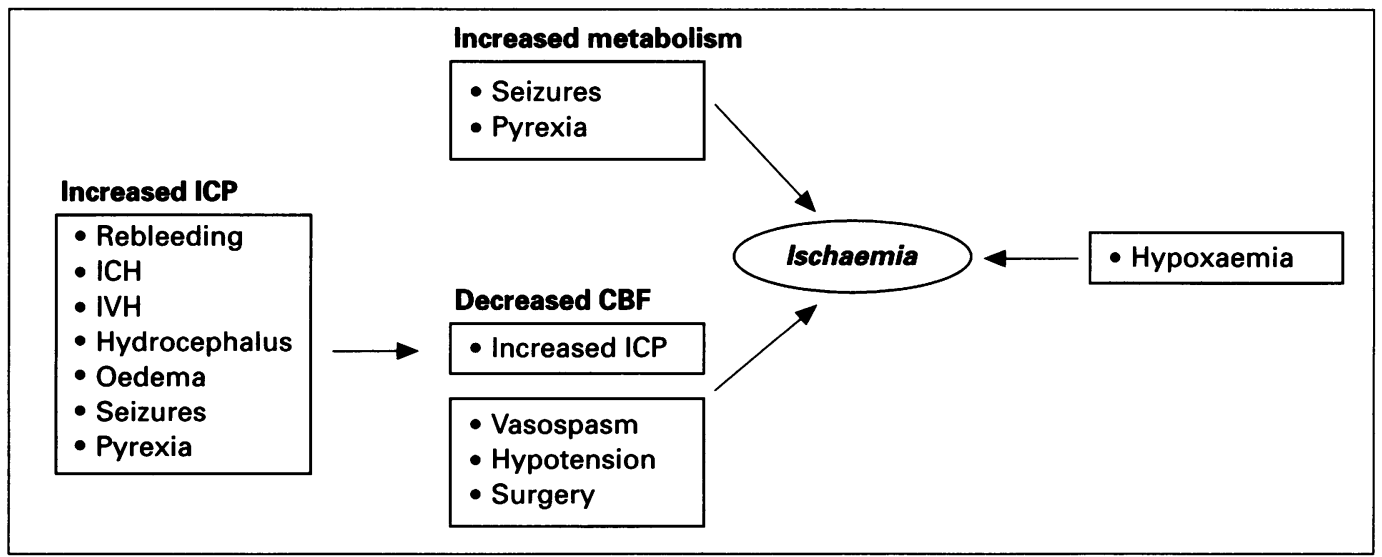

Figure 2 Complex interplay of insults responsible for secondary brain damage in subarachnoid haemorrhage. The sudden increase in intracranial pressure (ICP) caused by the aneurysm rupture renders the brain vulnerable to secondary insults. Secondary ischaemic brain damage is a result of insufficient cerebral blood flow (CBF) in relation to cerebral metabolism. Vasospasm, hypotension, and surgical trauma may all decrease the CBF. The CBF may also decrease because of an increase in ICP, which is in turn, caused by, for example, rebleeding, intracerebral (ICH), andlor intraventricular haematoma (IVH) and acute hydrocephalus. Cerebral metabolism is increased by infections, pyrexia, and seizures and this will, under certain conditions, increase the ICP and thereby also decrease CBF. Furthermore, an ischaemic lesion may become expansive and increase the ICP resulting in a further decrease in $C B F$. 
Hess grades I-III/IV-V (0-4/>4) complications, $P=0 \cdot 05)$. The mean number of complications in patients with a favourable outcome was $2 \cdot 1$ and in patients with an unfavourable outcome it was $4 \cdot 3\left(\chi^{2}\right.$ test, $0-4 />4$ complications: favourable/unfavourable outcome, $\mathrm{P}$ $=0.0008$, fig 1 ). The numbers for each individual avoidable factor were too small to permit detailed statistical analysis.

Multivariate analysis with the CT modified Hunt and Hess grade and the number of secondary brain insults during the first week as explanatory variables and favourable outcome as the dependent variable showed that the number of complications was a significant independent predictor of favourable outcome $(\beta=-0.38, \operatorname{SE}(\beta)=0.17, \mathrm{P}=0.03)$, whereas the CT modified Hunt and Hess grade did not reach significance $(\beta=-1 \cdot 2, \operatorname{SE}(\beta)=0 \cdot 81, P$ $=0 \cdot 14)$.

\section{Discussion}

The main objective of the neurointensive care unit is to counteract the development of secondary brain ischaemia by avoiding secondary insults that are likely to be responsible for ischaemic brain damage. The occurrence and impact of secondary insults in intensively managed patients with subarachnoid haemorrhage has, to our knowledge, not previously been analysed. This study shows that a substantial number of secondary events occur after subarachnoid haemorrhage despite the fact that the neurointensive care unit has the prerequisites to provide the intensive physiological monitoring and rapid intervention necessary to identify and treat such events. Corrie et al showed that about one third of the secondary insults in patients with head injury were missed by manual recording when compared with the findings from a computerised data collection system. ${ }^{11}$ Thus the number of secondary insults found in this study was probably underestimated, which further underlines the necessity to throw light on the occurrence of secondary insults after subarachnoid haemorrhage.

Defining different grades of secondary insults is an important issue. However, little is known about the relative influence of severity versus duration and the relative impact of different kinds of insults-for example, how does prolonged subfebrility compare with short periods of high fever and how does fever compare with hyperglycaemia. Based on our clinical experience and the preliminary results of this pilot study, more or less prolonged insults, such as pyrexia and hypotension, are scored maximally once a day, so as not to give such insults an unreasonable weight compared with more incidental insults such as herniation and rebleeding. Clinical signs of cerebral ischaemia and focal ischaemic lesions visible on CT were also registered as insults because they may also cause subsequent insults-for example, ischaemic oedema may increase ICP, which, in turn, may aggravate ischaemia, causing further oedema (fig 2). Systemic hypotension and increased ICP were registered separately despite their mathematical relation with CPP, because increased ICP and systemic hypotension seem to be independently related to outcome. ${ }^{3}$ Future efforts must concentrate on defining a grading system of secondary insults in subarachnoid haemorrhage similar to the Edinburgh University secondary insult grades (EUSIG) defined in patients with head injury.

This study suggests that the clinical outcome after aneurysmal subarachnoid haemorrhage is influenced by secondary insults that occur during neurointensive care. An important finding was that these secondary insults were probably not only a direct consequence of the subarachnoid haemorrhage, as the multivariate analyses showed that the number of secondary insults was significantly related to prognosis, whereas the CT modified Hunt and Hess grade did not add any significant prognostic information. It is reasonable to assume that the impact of a secondary insult is enhanced on the already stricken brain. In other words, the initial haemorrhage may render the brain vulnerable to secondary insults. Therefore, minor secondary insults may also be of importance. We think that this enhanced vulnerability caused by ictal events also prevails in patients who regain consciousness after ictus and may, at least in part, explain why some patients are more prone to develop delayed ischaemia and infarction than others. We have previously gained support for this hypothesis in an experimental study in rats which showed that the priming of the brain with a transient rise in ICP before a subsequent middle cerebral artery occlusion caused both increased infarct size and perifocal oedema. ${ }^{15}$

Awareness of the impact of secondary insults after subarachnoid haemorrhage may lead to further improvements in management. In our opinion, the importance of secondary insults in the development of delayed cerebral ischaemia and infarction has not been paid as much attention as it should, because of the preoccupation with vasospasm. To reduce the incidence of secondary ischaemic complications, more interest should probably be focused on early postictal events and on combating secondary insults. During recent years, we have the same emergency alertness in prehospital care, transportation of patients etc, as is now being used in the trauma care systems.

In conclusion, from this pilot study secondary insults after subarachnoid haemorrhage seem to be common. The number of insults was significantly lower in patients who had a more favourable outcome and probably had an independent impact on clinical outcome. Thus prospective studies should be initiated to monitor the insults more precisely and to define their impact on outcome after subarachnoid haemorrhage.

We thank neurointensive care nurse Birgitta Alverman for the follow up of the patients. This paper was presented in part at the 45th annual meeting of the Scandinavian Neurosurgical Society, Uppsala, 1993.

1 Reilly PL, Graham DI, Adams JH, Jennett B. Patients with head injury who talk and die. Lancet 1975;ii:375-7.

2 Rose J, Valtonen S, Jennett B. Avoidable factors contributing to death after head injury. $B M \mathcal{F} 1977 ; 2: 615-8$. 
3 Marmarou A, Anderson RL, Ward JD, et al. Impact of ICP instability and hypotension on outcome in patients with severe head trauma. F Neurosurg 1991;75:59-66.

4 Marshall LF, Toole BM, Bowers SA. The National Traumatic Coma Data Bank. Part 2: patients who talk and deteriorate: implications for treatment. $\mathcal{F}$ Neurosurg 1983;59:285-8.

5 Miller JD, Becker DP. Secondary insults to the injured brain. F $R$ Coll Surg Edinb 1982;27:292-8.

6 Miller JD, Sweet RC, Narayan R, Becker DP. Early insults to the injured brain. Fournal of the American Medical Association 1978;240:439-42.

7 Piek J, Chesnut RM, Marshall LF, et al. Extracranial complications of severe head injury. $f$ Neurosurg 1992;77: 901-7.

8 Sharples PM, Storey A, Aynsley-Green A, Eyre JA. Avoidable factors contributing to death of children with head injury. $B M \mathcal{F}$ 1990;300:87-91.

9 Gentleman D, Jennett B. Hazards of inter-hospital transfer of comatose head-injured patients. Lancet 1981;2:853-5.

10 Gentleman D, Jennett B. Audit of transfer of unconscious head-injured patients to a neurosurgical unit. Lancet 1990:335:330

11 Corrie J, Piper IR, Housley A. Microcomputer based data recording. Improving identification of secondary insults in head injured patients. British fournal of Intensive Care
1993;6:225-33.

12 Jones PA, Andrews PJD, Midgley S, et al. Measuring the burden of secondary insults in head-injured patients during intensive care. $\mathcal{F}$ Neurosurg Anesthesiol 1994;6:4-14.

13 Nornes $H$. The role of intracranial pressure in the arrest of haemorrhage in patients with ruptured intracranial aneurysm. F Neurosurg 1973;39:226-34.

14 Voldby B, Enevoldsen EM. Intracranial pressure changes following aneurysm rupture. Part 1: Clinical and angiographic correlations. $\mathcal{f}$ Neurosurg 1982;56:186-96.

15 Valtysson J, Jiang M, Persson L. Transient elevation of the intracranial pressure increases the infarct size and perifocal edema after subsequent middle cerebral artery occlusion in the rat. Neurosurgery 1992;30:887-90.

16 Hunt WE, Hess RM. Surgical risk as related to time of intervention in the repair of intracranial aneurysms. $f$ Neurosurg 1968;28:14-20.

17 Fisher CM, Kistler JP, Davis JM. Relation of cerebral vasospasm to subarachnoid haemorrhage visualized by computerized tomographic scanning. Neurosurgery 1980; 6:1-9.

18 Säveland H, Sonesson B, Ljunggren B, et al. Outcome evaluation following subarachnoid haemorrhage. $\mathcal{F}$ Neurosurg 1986;64:191-6.

19 Jennett $B$, Bond $M$. Assessment of outcome after severe brain damage: a practical scale. Lancet 1975;i:480-4. 